\title{
GOLD stage predicts thoracic aortic calcifications in patients with COPD
}

\author{
BERTRAM J. JOBST ${ }^{1-3}$, MICHAEL OWSIJEWITSCH ${ }^{1-3}$, HANS-ULRICH KAUCZOR $^{1-3}$, \\ JÜRGEN BIEDERER ${ }^{1,3,4}$, SEBASTIAN LEY ${ }^{1,5}$, NIKOLAUS BECKER ${ }^{6}$, ANNETTE KOPP-SCHNEIDER ${ }^{7}$, \\ STEFAN DELORME ${ }^{8}$, CLAUS PETER HEUSSEL $^{1-3}$, MICHAEL PUDERBACH $^{3,9}$, \\ MARK O. WIELPÜTZ ${ }^{1-3}$ and JULIA LEY-ZAPOROZHAN ${ }^{1,10}$
}

\begin{abstract}
${ }^{1}$ Department of Diagnostic and Interventional Radiology, University Hospital Heidelberg; ${ }^{2}$ Translational Lung Research Centre Heidelberg (TLRC), Member of The German Lung Research Centre (DZL), D-69120 Heidelberg; ${ }^{3}$ Department of

Diagnostic and Interventional Radiology with Nuclear Medicine, Thoraxklinik at University of Heidelberg,

D-69126 Heidelberg; ${ }^{4}$ Department of Radiology, Hospital Gross-Gerau, Darmstadt Private Practice for Radiology and Nuclear Medicine, D-64521 Gross-Gerau; ${ }^{5}$ Diagnostic and Interventional Radiology, Surgical Hospital Munich South,

D-81379 Munich; ${ }^{6}$ Division of Cancer Epidemiology; ${ }^{7}$ Division of Biostatistics, German Cancer Research Centre (DKFZ Heidelberg); ${ }^{8}$ Department of Radiology, German Cancer Research Centre (DKFZ Heidelberg), D-69120 Heidelberg;

${ }^{9}$ Department of Diagnostic and Interventional Radiology, Hufeland Hospital, D-99947 Bad Langensalza;

${ }^{10}$ Department of Radiology, Ludwig-Maximilians-University Hospital Munich, D-80337 Munich, Germany
\end{abstract}

Received June 20,2018; Accepted November 21, 2018

DOI: $10.3892 / \mathrm{etm} .2018 .7039$

\begin{abstract}
Although some of the associations between chronic obstructive pulmonary disease (COPD) and atherosclerosis are based on shared risk factors such as smoking, recent epidemiological evidence suggests that COPD is a risk factor for vascular disease due to systemic inflammation. The present study assessed the hypothesis that disease severity (as expressed by the GOLD stage) independently predicts the extent of vascular calcifications. A total of 160 smokers diagnosed with COPD (GOLD I-IV, 40 subjects of each GOLD stage) and 40 smokers at risk (GOLD 0; median age of 60 years old; Q1:56;Q3:65; 135 males and 65 females) underwent non-contrast, non-electrocardiography synchronized chest computerised tomography. The volume of thoracic aortic calcifications was quantified semi-automatically within a region from T1 through T12. Multiparametric associations with GOLD stage, smoking history, sex, age, body mass index and emphysema index were evaluated using generalized linear regression analysis. Thoracic aortic calcifications were highly prevalent in this cohort (187/200 subjects, 709 (Q1:109;Q3:2163) $\mathrm{mm}^{3}$ ). Analysis of variance on ranks
\end{abstract}

Correspondence to: Dr Bertram J. Jobst, Department of Diagnostic and Interventional Radiology, University Hospital Heidelberg, Neuenheimer Feld 110, D-69120 Heidelberg, Germany

E-mail: bertram.jobst@med.uni-heidelberg.de

Key words: chronic obstructive pulmonary disease, pulmonary emphysema, inflammation, multidetector-row computed tomography, atherosclerotic plaques, aortic diseases demonstrated a significant difference in calcium between different GOLD-stages as well as patients at risk of COPD $(\mathrm{F}=36.8, \mathrm{P}<0.001)$. In the multivariable analysis, GOLD-stages were indicated to be predictive of thoracic aortic calcifications $(\mathrm{P} \leq 0.0033)$ besides age $(\mathrm{P}<0.0001)$, while age appeared to be the strongest predictor. Other variables were not statistically linked to thoracic aortic calcifications in the multivariable model. COPD severity, as expressed by the GOLD-stage, is a significant predictor of thoracic aortic calcifications, independent of covariates such as age or tobacco consumption.

\section{Introduction}

Chronic obstructive pulmonary disease (COPD) is mainly caused by cigarette smoking and characterized by airflow limitation due to small airways disease and parenchymal destruction, mediated by chronic inflammation (1). COPD is a leading cause of morbidity and mortality worldwide and will become the fourth leading cause of death by 2030 (2). In addition, co-morbid conditions such as cardiovascular disease are found more frequently in COPD than would be expected by chance, even after adjustment for shared risk factors $(3,4)$. These comorbidities contribute to the deterioration of COPD, as well as mortality, especially due to cardiovascular disease (1). Although the exact mechanisms of interaction between COPD and vascular disease remain unclear, recent epidemiological evidence suggests that airway disease and emphysema are risk factors for vascular disease due to spillover of inflammatory cells and mediators from the lungs, resulting in systemic inflammation $(4,5)$. This is assumed to lead into persistent systemic endothelial dysfunction and subsequent atherosclerosis $(6,7)$. Hence, vascular disease in patients with COPD should be identified and managed where clinically appropriate (8). 
In general, quantitative coronary artery calcium scoring is a widely used approach to stratify the individual risk for cardiovascular events $(9,10)$. Thin-section non-contrast chest CT datasets potentially suitable for vascular calcium scoring are widely available in patients with COPD or smokers since imaging is frequently performed due to lung cancer screening, suspected pneumonia, or prior to endoscopic or surgical lung volume reduction. However, such CT data are usually non ECG (electrocardiography) synchronized, but motion correction based on ECG synchronization is regarded as a prerequisite for coronary artery calcium scoring since image artefacts due to cardiac motion can falsify quantitative calcium values of coronary arteries (8) even when using modern equipment such as 64-row MDCT scanners (11). Since motion artifacts are considerably lower in the thoracic aorta compared to coronary arteries, thoracic aortic calcium scoring based on non ECG-synchronized chest CT data provides potential surrogate parameters for the stratification of the vascular risk of individual COPD patients (11). The feasibility of thoracic aortic calcium scoring using low dose non ECG synchronized chest CT data has already been demonstrated (11-13). Moreover, thoracic aortic calcifications were shown to correlate with the extent of coronary artery calcifications (14) and to predict the development of calcified coronary plaques (15), cardiovascular events and especially non-cardiac events (13). Hence, thoracic aortic calcium scores represent important indicators of vascular health and provide appropriate parameters to clarify the question whether COPD is an independent risk factor for atherosclerosis. Previous CT based studies addressing associations between airflow limitation and vascular calcifications were mainly focused on coronary calcifications (16-19) mostly including subjects with mild airflow obstruction (17-19) and provided conflicting results. Hence, there is insufficient data on the relationship of clinically manifest or advanced COPD and the amount of vascular calcifications. Therefore, the study was aimed to involve smokers at risk of COPD as well as COPD patients with all degrees of disease severity, in order to test the hypothesis that the calcified plaque load of the thoracic aorta increases with COPD severity, expressed as GOLD stages (1), independent of shared risk factors such as tobacco use.

\section{Materials and methods}

Patient characteristics. A total of 200 subjects (further details on ethics approval and consent to participate are described in a separate section below) were enrolled into a retrospective study, in detail 160 smokers with clinically stable COPD (40 subjects of each GOLD stage I-IV) according to the GOLD criteria (1) and 40 heavy smokers at risk of COPD. The intention was to obtain a cohort with the complete spectrum of disease severity. Subjects were either participants of a population-based randomized lung cancer screening trial $(20,21)(142 / 200)$ or routine COPD patients (58/200). Key exclusion criteria were previous lung surgery, prior lung cancer, recent exacerbation of COPD ( $<4$ weeks, requiring antibiotic treatment), major pleural effusion, atelectasis, restrictive lung disease as well as previous surgery of the aorta. The majority of subjects was male (135/200). Ages ranged from 50 to 75 years. Median age was 60 (56-65) years, and median FEV1\% 67.4 (37.9-91.3). 199/200 patients were current or former heavy smokers.
Pulmonary function testing. Whole-body plethysmographic data (MasterScreen Body, E. Jaeger, Hoechberg, Germany) were acquired according to the guidelines of the European Respiratory Society and the American Thoracic Society (22), with the European Coal and Steel Community predicted values serving as standard of reference (23).

Multidetector computed tomography imaging. Volumetric non-enhanced non-ECG-synchronized thin-section MDCT datasets of the chest were acquired in caudocranial direction. Scans were obtained at end-inspiration breath-hold in supine position from the posterior costodiaphragmatic recess level to the superior thoracic aperture. Prior to scanning, all subjects received an instructed training to achieve full end-inspiratory breath-hold. CT acquisitions in lung cancer screening participants were performed with a 16 detector-row Toshiba Aquilion 16 (TOSHIBA Medical Systems GmbH, Zoetermeer, Netherlands) which is hereinafter referred to as 'scanner A', using a tube potential of $120 \mathrm{kVp}$, an effective tube current of $55 \mathrm{mAs}$, and a collimation of $16 \times 1.0 \mathrm{~mm}\left(\mathrm{CTDI}_{\mathrm{vol}}=5.1\right)$. Clinical indication based CT scans of routine COPD patients were acquired with a 4 detector-row Siemens Somatom Volume Zoom (Siemens AG, Helthcare Sector, Erlangen, Germany) which is hereinafter referred to as 'scanner B', applying a tube potential of $120 \mathrm{kVp}$, an effective tube current of $70 \mathrm{mAs}$, and a collimation of $4 \times 1.25 \mathrm{~mm}\left(\mathrm{CTDI}_{\mathrm{vol}}=6.3\right)$. Axial images for quantitative image evaluation were reconstructed using a medium-smooth convolution kernel (FC13/B35f) and a slice thickness of $3.0 \mathrm{~mm}$ with a reconstruction increment of $2.4 \mathrm{~mm}$. All image data were reconstructed with a field of view of 350-400 mm and a matrix of 512x512 voxels. Both scanners were calibrated for water at regular intervals (quarterly) and after major maintenance, and for air daily. All examinations were visually inspected by an experienced chest radiologist, validating sufficient inspiration, inclusion of all parts of the chest, and absence of significant motion artifacts or atelectasis.

Calcium scoring. After pseudonymization, image datasets were reviewed using a postprocessing workstation (Vitrea, Vital Images Inc., Minnetonka, MN, USA). The quantification of the total volume of thoracic aortic calcifications (TAC) was carried out on the thoracic aorta (ascending aorta including aortic bulb, aortic arch, and descending aorta down to the level of the base plate of chest vertebra 12). Within this region, semi-automated software-based calcium scoring was applied on axial CT slices using Vitrea Vscore (Vital Images Inc., Minnetonka, MN, USA). Calcified lesions exceeding a threshold of 130 Hounsfield units (HU) and 3 coherent voxels were highlighted automatically by a color overlay and verified manually. In this context, only vessel wall associated lesions were reported by the investigator. The TAC was summarized for each patient dataset. Using non ECG synchronized CT images, the volume score was favoured over the widespread Agatston score due to reduced susceptibility to motion artifacts (24). To ensure consistency of the image evaluation process, calcium scoring was performed by a single, experienced chest radiologist.

CT-based quantification of emphysema. The evaluation of the extent of pulmonary emphysema was based on the fully 
Table I. Summary of patient characteristics.

\begin{tabular}{lccccc}
\hline Variable & GOLD 0 & GOLD I & GOLD II & GOLD III & GOLD IV \\
\hline Patients (n) & 40 & 40 & 40 & 40 & 40 \\
Scanner A/B (n) & $40 / 0$ & $40 / 0$ & $40 / 0$ & $14 / 26$ & $8 / 32$ \\
Male/female (n) & $27 / 13$ & $26 / 14$ & $30 / 10$ & $21 / 19$ & $26 / 14$ \\
Age (y) & $58(54-63)$ & $57(53-63)$ & $59(56-65)$ & $64(59-67)^{\mathrm{a}, \mathrm{b}}$ & $61(57-67)^{\mathrm{b}}$ \\
FEV1\% & $94.8(89.2-103.6)$ & $92.2(86.0-98.1)$ & $69.0(62.5-74.3)$ & $40.9(36.0-44.0)$ & $25.9(21.5-31.5)$ \\
Pack-years (n) & $37(32-54)$ & $33(26-45)$ & $44(33-63)$ & $41(30-60)$ & $40(30-60)$ \\
BMI $\left(\mathrm{kg} / \mathrm{m}^{2}\right)$ & $27.3(24.3-29.8)$ & $24.8(22.1-26.5)^{\mathrm{a}}$ & $25.7(23.9-28.4)$ & $25.2(23.4-28.0)$ & $24.0(19.8-25.9)^{\mathrm{a}, \mathrm{c}}$ \\
LAA\% & $5.6(1.5-9.6)$ & $9.5(4.5-18.2)$ & $12.3(4.8-26.0)$ & $43.7(35.0-53.1)^{\mathrm{a}-\mathrm{c}}$ & $49.6(40.1-59.1)^{\mathrm{a}-\mathrm{c}}$ \\
TAC $\left(\mathrm{mm}^{3}\right)$ & $239(25-1119)$ & $141(30-748)$ & $789(196-1861)^{\mathrm{b}}$ & $1,868(585-4097)^{\mathrm{a}, \mathrm{b}}$ & $1,385(431-3005)^{\mathrm{a}, \mathrm{b}}$ \\
\hline
\end{tabular}

All data reported as medians and interquartile range. FEV1\%, forced expiratory volume in 1 second percentage predicted; TAC, total volume of thoracic aortic calcifications; Scanner A, Toshiba Aquilion 16; scanner B, Siemens Somatom Volume Zoom 4; BMI, body mass index; LAA\%, low attenuation areas in percent. ${ }^{\mathrm{a}} \mathrm{P}<0.05$ vs. at risk, ${ }^{\mathrm{b}} \mathrm{P}<0.05$ vs. GOLD I, ${ }^{\mathrm{c}} \mathrm{P}<0.05$ vs. GOLD II.

automatic quantitative in-house software YACTA (v1.0.9.38) and MeVisPULMO 3D, (Fraunhofer MEVIS, Bremen, Germany), calculating the relative volume of low attenuation areas (LAA\%, density threshold $-950 \mathrm{HU}$ ) in relation to the total lung volume. The exact steps of structural lung segmentation and emphysema quantification are described and validated in detail elsewhere (25-27).

Statistical analysis. Calculations were performed using IBM SPSS Statistics v19 (IBM Deutschland GmbH, Ehingen, Germany), SigmaPlot v12.5 (Systat Software Inc., San Jose, CA, USA) and SAS v9.4 (SAS Institute, Cary, North Carolina, USA). Data are expressed as frequencies (percentages) or as medians with interquartile range (Q1-Q3). Intergroup comparison was performed using the independent samples t-test or one-way ANOVA with appropriate post-hoc testing for normal distributions. Intergroup comparison was performed by Mann-Whitney U test or Kruskal-Wallis one-way ANOVA on ranks with appropriate post-hoc testing for skewed distributions. Intergroup comparison for categorical data was based on Fisher's exact test. Multivariable analysis was performed using multivariable linear regression with $\log 10$-transformed TAC as a dependent variable and age, GOLD stage, BMI, sex and LAA\% as independent variables. Based on this multivariable model, it was assessed whether age, GOLD stage, BMI, sex and LAA\% could independently predict the extent of log10TAC.

Collinearity between dependent variables (age, GOLD stage, BMI, sex, LAA\%, pack years) was tested using Pearson product-moment correlation coefficient for normal and Spearman's rank correlation coefficient for skewed distributions. Multiple testing was compensated for by the Bonferroni-Holm method (28).

\section{Results}

Thoracic aortic calcifications were present in 187/200 subjects. Median TAC was 709 (109-2163) $\mathrm{mm}^{3}$ for the entire population and increased from GOLD I to GOLD III without further increase towards GOLD IV (Table I). Interestingly, smokers at risk of COPD (GOLD 0) showed slightly higher TAC than
GOLD I subjects. Kruskal-Wallis one-way ANOVA on ranks showed a significant difference in overall calcium between subjects with different GOLD-stages as well as patients at risk $(\mathrm{F}=36.8 \mathrm{P}<0.001)$. Subjects with GOLD stage I and subjects at risk of COPD showed significantly lower TAC values in relation to GOLD stages III and IV (each $\mathrm{P}<0.05$ ). Subjects with GOLD stage I had significantly lower TAC compared to GOLD II $(\mathrm{P}<0.05)$ (Table I).

Almost all subjects were current or former heavy smokers with similarly high amount of packyears 40 (30-60), which did not differ significantly between groups of different disease severity $(\mathrm{F}=6.7, \mathrm{P}=0.15)$. The distribution of male and female participants did not show significant variability across the patient groups $(\mathrm{P}=0.06-1.00)$. The median BMI of 25.2 (23.0-28.1) of the cohort was slightly elevated. One-way ANOVA showed a significant difference in BMI between the respective groups $(\mathrm{F}=6.0, \mathrm{P}<0.001)$. Holm-Sidak post-hoc test indicated that the BMI of subjects with GOLD stage IV (24.0 (19.8-25.9)) was significantly lower compared to subjects at risk of COPD (27.3 (24.3-29.8)) $(\mathrm{P}<0.001)$ and subjects with GOLD stage II (25.7 (23.9-28.4)) $(\mathrm{P}<0.05$; Table I). Kruskal-Wallis One-way ANOVA on ranks showed a significant difference in LAA\% between the respective groups $(\mathrm{P}<0.001)$ with a steady increase from subjects at risk of COPD towards GOLD stage IV (Table I). Dunn's post hoc test revealed a significant difference between GOLD stage III or IV in relation to GOLD stages I-II as well as subjects at risk (each $\mathrm{P}<0.05)$. A significant difference between GOLD stage III and IV could not be observed (Table I).

The multivariable regression model (Table II) revealed that GOLD-stage was a significant predictor of $\log 10 \mathrm{TAC}$ besides age, each independent from other risk factors of atherosclerosis, and age appeared to be the strongest predictor (Table II). BMI, sex, pack years, and LAA\% were not statistically linked to the extent of TAC (Table II). The coefficient of determination $\left(\mathrm{R}^{2}\right)$ of the regression model was 0.288 , indicating that $28.8 \%$ of the variability of $\log 10 \mathrm{TAC}$ can be explained by age and GOLD stage.

Since CT images of subjects attributed with GOLD stages III and IV were acquired with two different CT 
Table II. Predictors of thoracic aortic calcifications based on multivariable linear regression analysis.

\begin{tabular}{lrr}
\hline Predictor & Estimate & P-value \\
\hline Age & 0.099 & $<0.0001$ \\
GOLD 0 & -4.933 & $<0.0001$ \\
GOLD I & -4.879 & $<0.0001$ \\
GOLD II & -4.204 & 0.0004 \\
GOLD III & -4.018 & 0.0014 \\
GOLD IV & -3.581 & 0.0033 \\
BMI & 0.038 & 0.1423 \\
LAA\% & -0.009 & 0.2183 \\
Sex & 0.193 & 0.3194 \\
Pack-years & 0.001 & 0.8199 \\
\hline
\end{tabular}

Estimate, parameter estimate; BMI, body mass index; LAA\%, low attenuation areas in percent.

scanners, the presence of substantial inter-scanner variability concerning TAC and LAA\% had to be ruled out: Importantly, no significant difference in TAC was observed for scanner A (1371(246-3002) $\left.\mathrm{mm}^{3}\right)$ vs. scanner B (2310(616-4751) $\left.\mathrm{mm}^{3}\right)$ $(\mathrm{P}=0.38)$ in the subgroup with GOLD stage III or for scanner A (1280(227-3826) $\left.\mathrm{mm}^{3}\right)$ vs. scanner B (1402(436-2755) $\left.\mathrm{mm}^{3}\right)$ in the subgroup with GOLD stage IV $(\mathrm{P}=0.90)$. By analogy, no significant difference in LAA\% was observed for scanner A (40.2(33.7-58.9)\%) vs. scanner B (44.3(34.6-49.6)\%) $(\mathrm{P}=0.74)$ within GOLD stage III and for scanner A (52.1(45.2-73.5)\%) vs. scanner B (47.9(39.9-58.2)\%) in GOLD stage IV ( $\mathrm{P}=0.06)$.

\section{Discussion}

The study was conducted to elucidate associations between thoracic aortic calcifications, COPD severity (at risk of COPD, GOLD I-IV) and shared risk factors to test the hypothesis that COPD on its own represents an independent predictor for atherosclerosis.

Unsurprisingly, thoracic aortic calcifications were highly prevalent in this cohort (187/200 subjects) with an average plaque volume of $709(109-2163) \mathrm{mm}^{3}$. Multivariable regression analysis revealed that increasing COPD severity (as expressed by the GOLD stage) was associated with aggravation of the thoracic aortic calcium load, independently of tobacco use or other shared risk factors. Besides, age was also shown to be a significant predictor of thoracic aortic calcifications. All other variables (BMI, sex, pack-years, LAA\%) were not statistically linked to thoracic aortic calcifications in the multivariable analysis.

As mentioned in the introduction section, there is limited information on the relationship between COPD severity and the amount of vascular calcifications. Using multiple logistic regression analysis, Chae et al (29) demonstrated age and FEV1/FVC ratio to be independent predictors of the amount of intrathoracic arterial calcifications (Agatston score $>1,000$ ) in 206 subjects diagnosed with (mean FEV1\%=53.5) or at risk of COPD. Although the proportional distribution of GOLD stages in this cohort is not reported, the findings of Chae et al (29) are in conformity with our results. By analogy to our study, other risk factors such as smoking history, BMI or diffusing capacity for carbon monoxide (DLCO) were not predictive of thoracic arterial calcifications in their multivariable regression model. Using a multivariable regression in a similar cohort with 240 subjects, Dransfield et al (30) found independent associations between the thoracic aortic calcium load (assessed by Agatston score) and age as well as quantitative CT-based emphysema metrics, but not with airflow limitation in terms of FEV1/FCV ratio. The role of FEV1\% or GOLD stage was not investigated in their multivariable model. Williams et al (16), addressed coronary artery calcifications and demonstrated that patients with COPD had higher coronary calcium scores than smokers and non-smoking controls in a cohort of 672 subjects with COPD, 199 smokers without airflow obstruction and 71 non-smokers. Moreover, they demonstrated that coronary calcium scores were increased in subjects who died until the 3-year follow-up compared to those who survived.

Since atherosclerosis is well known to be an age-related disease, the increase of vascular calcifications with age as observed in the present study and other studies (30) is not surprising.

An increased BMI is a well-known risk factor for vascular calcifications (31) and BMI values are known to be slightly elevated in subjects with early stages of COPD (32). In advanced stages, BMI values decrease (32) due to cachexia and muscle wasting. In the present study, subjects at risk of COPD as well as in GOLD stages II and III showed slightly increased BMI, whereas subjects with GOLD stage 4 showed normal BMI, which was significantly lower compared to smokers at risk and GOLD stage II patients. However, in the present study BMI was not linked to the aortic plaque load as demonstrated by multivariable analysis.

It has been known for many decades, that vascular diseases are more frequent in men than in women, amongst others due to a love for rich food, alcohol, and tobacco (33). However, the gender was not identified as a predictor of atherosclerosis in our study cohort after adjustment for other risk factors.

It can be assumed that in this cohort, smoking history was not identified as a predictor of aortic calcium, since almost all subjects (199/200) were current or former heavy smokers, with almost matching amount of pack-years 40 (30-60), which did not differ significantly between different GOLD stages. By analogy, Dransfield et al (30) reported that the amount of pack years did not show significant associations with aortic calcium after adjusting for covariates in a cohort of heavy smokers diagnosed with or at risk of COPD. However, the importance of cigarette smoking in the development of COPD and atherosclerosis has been extensively described before $(34,35)$.

Although LAA\% gradually increased from subjects at risk of COPD towards GOLD stage IV, it was not a significant predictor of aortic calcifications. By analogy, Williams et al (16) demonstrated that the calcium load of coronary arteries was higher in subjects with COPD compared to normal smokers and non-smokers and correlated with age, pack years, and 6-minute walk test, but not with the extent of emphysema or FEV1\%. Discrepancies concerning the association between emphysema and vascular calcifications may be explained by the fact that COPD is a heterogeneous disease 
with different phenotypes, such as airway predominant and emphysema predominant phenotypes $(36,37)$, which were not taken into account in the present study. Different CT equipment is known to potentially influence the quantitative evaluation of lung parenchyma (38) and vascular calcifications (39) in the context of multicentre imaging. However, quantitative aortic calcium and LAA\% values were not significantly different between the two CT devices used in this study, implying that scanner-related effects on quantitative CT analyses can be regarded negligible in this study. Of note, FEV1\% as a variable was excluded from the multivariable model due to high collinearity with the variable 'GOLD stage'.

For the sake of completeness, it has to be mentioned that TAC increased from GOLD I to GOLD III without further increase towards GOLD IV. Instead, TAC values showed a moderate decrease in GOLD IV, which may be partly explained by the fact that the median age was lower in GOLD IV 61 (57-67) than in the GOLD III group 64 (59-67). This difference was not statistically significant, but the multivariable analysis demonstrated age to be a strong predictor of TAC. Besides, the lower TAC in GOLD IV may be partly based on factors which were not considered in this study, such as vasoprotective medication or genetic disposition. In this context, the GOLD IV group showed a higher degree of COPD severity at younger age than GOLD III, although the amount of pack years being very similar, possibly reflecting different genetic disposition for smoke-induced lung disease and maybe vascular disease. The fast progression of COPD of the GOLD IV group may have driven early therapy onset (earlier in life compared to GOLD III), possibly including vasoprotective medication.

The awareness that COPD is independently linked to atherosclerosis bears relevance, since vascular disease significantly contributes to deterioration and mortality in COPD patients (1). The quantification of thoracic aortic calcium has already been demonstrated to provide a surrogate parameter for the vascular risk of COPD patients (11). In this context, data from the Multi-Ethnic Study of Atherosclerosis (MESA) demonstrated excellent correlation between thoracic aortic and coronary calcifications (14). In a large lung cancer screening population, the aortic calcium load has been shown to be rather associated with non-cardiac events such as stroke and peripheral artery disease (13). Interestingly, coronary calcium scores seemed to correlate better with all-cause mortality (13). Since non ECG synchronized chest CT data are widely available in subjects with COPD through clinical imaging or recently established lung cancer screening programs, they provide a huge potential for vascular risk stratification and optimized treatment of clinically relevant vascular comorbidity, thus preventing vascular events and reducing mortality in COPD patients (40). While coronary calcium scoring on non ECG synchronized CT data is discussed controversely due to motion artifacts $(8,11)$, the calcium load of the thoracic aorta can be effectively quantified on non ECG synchronized chest CT data (11-13). Consequently, calcium scoring on non ECG synchronized CT data helps to gather more information about the patient, thus making the most out of radiation-based images. While population-based reference values of the vascular calcium load already exist for coronary artery calcium scoring and allow to estimate the individual risk for vascular events and to optimize the treatment, such reference values have not been established yet for the thoracic aorta.

A potential limitation of the present study is that the calcium load was not normalized in relation to the body size of the subjects. Moreover, several factors potentially influencing the vascular plaque load such as diabetes, triglyceride levels, genetic disposition or vasoprotective medication were not considered in the present study. The present study only addressed calcified plaques. However, noncalcified plaques are of high relevance in vascular disease as demonstrated by Büyükterzi et al (41) in a study with 288 subjects lacking calcified plaques in the coronary arteries, with $17.4 \%$ of their patients showing substantial coronary soft plaques, and $2.1 \%$ showing significant stenosis on coronary computed tomography. Besides, cardiovascular diseases reflect a broad spectrum of disease entities, such as cardiac diseases of different origin, and atherosclerosis with its diverse manifestations, while these entities are often linked or have common risk factors (42-44). Cardiovascular disease was not excluded from our study population since the study design required the investigation of aortic calcifications. In this context, aortic calcifications themselves are a marker or surrogate parameter for the extent of atherosclerosis which is strongly associated with other entities of cardiovascular disease $(13,15)$. Therefore, excluding subjects with cardiovascular disease would have resulted in significant selection bias. Consequently, caution should be taken when interpreting the findings. However, since we did not investigate calcium scores of coronary arteries, the presence of coronary artery stents (potentially mimicking calcified plaques) in subjects with coronary artery disease did not affect our results. It is also unknown whether aortic aneurysms or ectasia have impact on aortic calcium scores. However, aortic aneurysms were absent in our study population.

In brief, including subjects with the entire spectrum of disease severity and also subjects at risk of COPD, we could demonstrate that the severity of COPD (GOLD stage) is a significant predictor of thoracic aortic calcifications, independent from other risk factors such as age or cigarette smoking, underlining the role of COPD as a risk factor for atherosclerosis as suspected by other studies with different design. Besides, age was identified to be a strong predictor of thoracic aortic calcifications. Thoracic aortic calcium scoring can be accurately performed on non-ECG-synchronized CT images, which are frequently performed in COPD patients due to various clinical indications. Considering the fact that vascular disease significantly contributes to deterioration and mortality in COPD patients, calcium scoring performed on non-ECG-synchronized CT images may help to stratify the vascular risk and optimize the vascular treatment of COPD patients.

\section{Acknowledgements}

Not applicable.

\section{Funding}

The present study has been supported by the Competence Network Asthma/COPD funded by the German Federal Ministry of Education and Research (FKZ 01GI0884). The German Lung Cancer Screening Intervention Trial (LUSI) 
was supported by the German Research Foundation (DFG: BE 2486 2-1 (2007-2010) and BE 2486 2-2 (2010-2013)) and the Dietmar-Hopp-Foundation (2007-2010).

\section{Availability of data and materials}

All data generated or analyzed during the present study are included in this published article.

\section{Authors' contributions}

BJJ, MO, HUK, JB, SL, NB, AKS, SD, CPH, MP, MOW and JLZ conceived and designed the experiments. BJJ, MO, HUK, SL, NB, SD, CPH, MP, MOW and JLZ were responsible for patient enrollment. BJJ performed semi-automatic calcium scoring based on the CT images. BJJ, MO, HUK, JB, SL, $\mathrm{NB}, \mathrm{AKS}, \mathrm{SD}, \mathrm{CPH}, \mathrm{MP}, \mathrm{MOW}$ and JLZ gathered all other patient data. BJJ, MO, HUK, JB, SL, NB, AKS, SD, CPH, MP, MOW and JLZ analyzed and interpreted the patient data. BJJ, MO, HUK, JB, SL, NB, AKS, SD, CPH, MP, MOW and JLZ contributed to writing the manuscript. All authors read and approved the final manuscript.

\section{Ethics approval and consent to participate}

The present study was approved by the Ethics Committee of the Medical Faculty of the University of Heidelberg, Germany (S-073/2001, S-646/2016). All participants gave written informed consent for CT examination, data collection and further data processing. The work was carried out in accordance with The Code of Ethics of the World Medical Association (Declaration of Helsinki).

\section{Patient consent for publication}

Not applicable.

\section{Competing interests}

The authors declare that they have no competing interests.

\section{References}

1. Vestbo J,Hurd SS, Agustí AG,Jones PW, Vogelmeier C,Anzueto A, Barnes PJ, Fabbri LM, Martinez FJ, Nishimura M, et al: Global strategy for the diagnosis, management, and prevention of chronic obstructive pulmonary disease: GOLD executive summary. Am J Respir Crit Care Med 187: 347-365, 2013.

2. Mathers CD and Loncar D: Projections of global mortality and burden of disease from 2002 to 2030. PLoS Med 3: e442, 2006.

3. Sin DD, Wu L and Man SF: The relationship between reduced lung function and cardiovascular mortality: A population-based study and a systematic review of the literature. Chest 127: 1952-1959, 2005.

4. Decramer M, Rennard S, Troosters T, Mapel DW, Giardino N, Mannino D, Wouters E, Sethi S and Cooper CB: COPD as a lung disease with systemic consequences-clinical impact, mechanisms, and potential for early intervention. COPD 5: 235-256, 2008.

5. Tan SL and Wood AM: Chronic obstructive pulmonary disease and comorbidity: A review and consideration of pathophysiology. Panminerva Med 51: 81-93, 2009.

6. Vukic Dugac A, Ruzic A, Samarzija M, Badovinac S, Kehler T and Jakopovic M: Persistent endothelial dysfunction turns the frequent exacerbator COPD from respiratory disorder into a progressive pulmonary and systemic vascular disease. Med Hypotheses 84: 155-158, 2015.
7. Eickhoff P, Valipour A, Kiss D, Schreder M, Cekici L, Geyer K, Kohansal R and Burghuber OC: Determinants of systemic vascular function in patients with stable chronic obstructive pulmonary disease. Am J Respir Crit Care Med 178: 1211-1218, 2008.

8. Boschetto P, Beghé B, Fabbri LM and Ceconi C: Link between chronic obstructive pulmonary disease and coronary artery disease: Implication for clinical practice. Respirology 17: 422-431, 2012.

9. Shaw LJ, Raggi P, Schisterman E, Berman DS and Callister TQ: Prognostic value of cardiac risk factors and coronary artery calcium screening for all-cause mortality. Radiology 228: 826-833, 2003.

10. Greenland P, LaBree L, Azen SP, Doherty TM and Detrano RC: Coronary artery calcium score combined with Framingham score for risk prediction in asymptomatic individuals. JAMA 291: 210-215, 2004.

11. Budoff MJ, Nasir K, Kinney GL, Hokanson JE, Barr RG, Steiner R, Nath H, Lopez-Garcia C, Black-Shinn J and Casaburi R: Coronary artery and thoracic calcium on noncontrast thoracic CT scans: Comparison of ungated and gated examinations in patients from the COPD Gene cohort. J Cardiovasc Comput Tomogr 5: 113-118, 2011.

12. Isgum I, Rutten A, Prokop M, Staring M, Klein S, Pluim JP, Viergever MA and van Ginneken B: Automated aortic calcium scoring on low-dose chest computed tomography. Med Phys 37: 714-723, 2010.

13. Jacobs PC, Prokop M, van der Graaf Y, Gondrie MJ, Janssen KJ, de Koning HJ, Isgum I, van Klaveren RJ, Oudkerk M, van Ginneken B and Mali WP: Comparing coronary artery calcium and thoracic aorta calcium for prediction of all-cause mortality and cardiovascular events on low-dose non-gated computed tomography in a high-risk population of heavy smokers. Atherosclerosis 209: 455-462, 2010.

14. Rivera JJ, Nasir K, Katz R, Takasu J, Allison M, Wong ND, Barr RG, Carr JJ, Blumenthal RS and Budoff MJ: Relationship of thoracic aortic calcium to coronary calcium and its progression (from the Multi-Ethnic Study of Atherosclerosis [MESA]). Am J Cardiol 103: 1562-1567, 2009.

15. Brodov Y, Gransar H, Rozanski A, Hayes SW, Friedman JD, Thomson LE, Dey D, Slomka PJ, Min JK, Shaw LJ, et al: Extensive thoracic aortic calcification is an independent predictor of development of coronary artery calcium among individuals with coronary artery calcium score of zero. Atherosclerosis 238: 4-8, 2015.

16. Williams MC, Murchison JT, Edwards LD, Agustí A, Bakke P, Calverley PM, Celli B, Coxson HO, Crim C, Lomas DA, et al: Coronary artery calcification is increased in patients with COPD and associated with increased morbidity and mortality. Thorax 69: 718-723, 2014.

17. Barr RG, Ahmed FS, Carr JJ, Hoffman EA, Jiang R, Kawut SM and Watson K: Subclinical atherosclerosis, airflow obstruction and emphysema: The MESA lung study. Eur Respir J 39: 846-854, 2012.

18. Park HY, Lim SY, Hwang JH, Choi JH, Koh WJ, Sung J, Suh GY, Chung MP, Kim H, Choe YH, et al: Lung function, coronary artery calcification, and metabolic syndrome in 4905 Korean males. Respir Med 104: 1326-1335, 2010.

19. Sverzellati N, Cademartiri F, Bravi F, Martini C, Gira FA, Maffei E, Marchianò A, La Vecchia C, De Filippo M, Kuhnigk JM, et al: Relationship and prognostic value of modified coronary artery calcium score, FEV1, and emphysema in lung cancer screening population: The MILD trial. Radiology 262: 460-467, 2012.

20. Becker N, Motsch E, Gross ML, Eigentopf A, Heussel CP, Dienemann H, Schnabel PA, Pilz L, Eichinger M, Optazaite DE, et al: Randomized study on early detection of lung cancer with MSCT in Germany: Study design and results of the first screening round. J Cancer Res Clin Oncol 138: 1475-1486, 2012.

21. Becker N, Motsch E, Gross ML, Eigentopf A, Heussel CP, Dienemann H, Schnabel PA, Eichinger M, Optazaite DE, Puderbach M, et al: Randomized study on early detection of lung cancer with MSCT in Germany: Results of the first 3 years of follow-up after randomization. J Thorac Oncol 10: 890-896, 2015.

22. Miller MR, Hankinson J, Brusasco V, Burgos F, Casaburi R, Coates A, Crapo R, Enright P, van der Grinten CP, Gustafsson P, et al: Standardisation of spirometry. Eur Respir J 26: 319-338, 2005.

23. Quanjer PH, Tammeling GJ, Cotes JE, Pedersen OF, Peslin R and Yernault JC: Lung volumes and forced ventilatory flows. Report Working Party Standardization of Lung Function Tests, European Community for Steel and Coal. Official Statement of the European Respiratory Society. Eur Respir J Suppl 16: 5-40, 1993. 
24. Groen JM, Greuter MJ, Vliegenthart R, Suess C, Schmidt B, Zijlstra F and Oudkerk M: Calcium scoring using 64-slice MDCT, dual source CT and EBT: A comparative phantom study. Int J Cardiovasc Imaging 24: 547-556, 2008.

25. Heussel CP, Herth FJ, Kappes J, Hantusch R, Hartlieb S, Weinheimer O, Kauczor HU and Eberhardt R: Fully automatic quantitative assessment of emphysema in computed tomography: Comparison with pulmonary function testing and normal values. Eur Radiol 19: 2391-2402, 2009.

26. Weinheimer O, Achenbach T, Heussel CP and Düber C: Automatic lung segmentation in MDCT images. Res Gate: pp241-255, 2011

27. Owsijewitsch M, Ley-Zaporozhan J, Kuhnigk JM Kopp-Schneider A, Eberhardt R, Eichinger M, Heussel CP, Kauczor HU and Ley S: Quantitative emphysema distribution in anatomic and non-anatomic lung regions. COPD 12: 257-266, 2015

28. Holm S: A simple sequentially rejective multiple test procedure. Scandinavian J Stat 6: pp65-70, 1979.

29. Chae EJ, Seo JB, Oh YM, Lee JS, Jung Y and Lee SD: Severity of systemic calcified atherosclerosis is associated with airflow limitation and emphysema. J Comput Assist Tomogr 37: 743-749, 2013.

30. Dransfield MT, Huang F, Nath H, Singh SP, Bailey WC and Washko GR: CT emphysema predicts thoracic aortic calcification in smokers with and without COPD. COPD 7: 404-410, 2010

31. Mahoney LT, Burns TL, Stanford W, Thompson BH, Witt JD, Rost CA and Lauer RM: Usefulness of the Framingham risk score and body mass index to predict early coronary artery calcium in young adults (Muscatine Study). Am J Cardiol 88 : 509-515, 2001

32. Agusti A, Calverley PM, Celli B, Coxson HO, Edwards LD, Lomas DA, MacNee W, Miller BE, Rennard S, Silverman EK, et al: Characterisation of COPD heterogeneity in the ECLIPSE cohort. Respir Res 11: 122, 2010.

33. Dock W: Why are men's coronary arteries so sclerotic. J Am Med Assoc 170: 152-156, 1959.

34. Sackett DL, Gibson RW, Bross ID and Pickren JW: Relation between aortic atherosclerosis and the use of cigarettes and alcohol. An autopsy study. N Engl J Med 279: 1413-1420, 1968.
35. Doll R, Peto R, Boreham J and Sutherland I: Mortality in relation to smoking: 50 years' observations on male British doctors. BMJ 328: 1519, 2004

36. Ley-Zaporozhan $\mathbf{J}$ and van Beek EJ: Imaging phenotypes of chronic obstructive pulmonary disease. J Magn Reson Imaging 32: 1340-1352, 2010

37. Galbán CJ, Han MK, Boes JL, Chughtai KA, Meyer CR Johnson TD, Galbán S, Rehemtulla A, Kazerooni EA, Martinez FJ and Ross BD: Computed tomography-based biomarker provides unique signature for diagnosis of COPD phenotypes and disease progression. Nat Med 18: 1711-1715, 2012.

38. Coxson HO: Sources of variation in quantitative computed tomography of the lung. J Thorac Imaging 28: 272-279, 2013

39. Willemink MJ, Vliegenthart R, Takx RA, Leiner T, Budde RP, Bleys RL, Das M, Wildberger JE, Prokop M, Buls N, et al: Coronary artery calcification scoring with state-of-the-art CT scanners from different vendors has substantial effect on risk classification. Radiology 273: 695-702, 2014.

40. Mets OM, de Jong PA and Prokop M: Computed tomographic screening for lung cancer: An opportunity to evaluate other diseases. JAMA 308: 1433-1434, 2012.

41. Büyükterzi M, Türkvatan A and Büyükterzi Z: Frequency and extent of coronary atherosclerotic plaques in patients with a coronary artery calcium score of zero: Assessment with CT angiography. Diagn Interv Radiol 19: 111-118, 2013.

42. Vogelmeier CF, Criner GJ, Martínez FJ, Anzueto A, Barnes PJ, Bourbeau J, Celli BR, Chen R, Decramer M, Fabbri LM, et al: Global strategy for the diagnosis, management, and prevention of chronic obstructive lung disease 2017 report. GOLD executive summary. Arch Bronconeumol 53: 128-149, 2017.

43. GBD 2013 Mortality and Causes of Death Collaborators: Global, regional, and national age-sex specific all-cause and cause-specific mortality for 240 causes of death, 1990-2013: A systematic analysis for the Global Burden of Disease Study 2013. Lancet 385: 117-171, 2015.

44. Mendis S, Puska P and Norrving B; World Health Organization, World Heart Federation: Global atlas on cardiovascular disease prevention and control. World Health Organization, Geneva, 2011. 